\title{
Influência do sistema de criação nos parâmetros comportamentais de duas linhagens de poedeiras submetidas a duas condições ambientais
}

\author{
Iran José Oliveira da Silva ${ }^{1}$, José Antonio Delfino Barbosa Filho ${ }^{2}$, Marco Aurélio \\ Neves da Silva ${ }^{3}$, Sônia Maria de Stefano Piedade ${ }^{4}$
}

\footnotetext{
${ }^{1}$ Departamento de Engenharia Rural - NUPEA/ESALQ/USP - Piracicaba - SP - Brasil.

2 Departamento de Física do Ambiente Agrícola - NUPEA/ ESALQ/USP - Piracicaba - SP - Brasil.

3 Doutorando - Departamento de Genética - ESALQ/USP - Piracicaba - SP - Brasil.

${ }^{4}$ Departamento de Ciência Exatas - ESALQ/USP - Piracicaba - SP - Brasil.
}

RESUMO - Este estudo foi realizado com os objetivos de avaliar o comportamento e o bem-estar de aves em um sistema de criação em cama e ninho, em comparação a um sistema de criação convencional (bateria de gaiolas), e estudar a influência das condições ambientais (estresse e conforto) no comportamento de poedeiras. Dois grupos de dez aves (dez Hy-line W36 e dez Hy-line Brown) em início de postura foram submetidos a duas condições ambientais $\left(26^{\circ} \mathrm{C}\right.$ e $60 \%$ UR e $35^{\circ} \mathrm{C}$ e $\left.70 \% \mathrm{UR}\right)$ e dois sistemas de criação (bateria de gaiolas e cama) durante duas semanas consecutivas. Durante o período de avaliação, um sistema de câmeras de vídeo instaladas no teto da câmara climática registrava o comportamento das aves. Os parâmetros comportamentais foram avaliados durante 2 horas no período da manhã e 2 horas no período da tarde, com gravações feitas a cada segundo. Para melhor avaliação individual do comportamento, as aves foram marcadas com tinta atóxica. Pela análise do comportamento, verificou-se que o sistema de criação em cama propiciou a expressão de todos os comportamentos naturais e de conforto das aves, fornecendo melhores condições de bem-estar. No sistema de criação convencional (gaiolas), mesmo sem nenhuma condição, as aves tentaram executar seus hábitos naturais e, certamente, a impossibilidade de expressarem esses comportamentos agravou o estresse provocado por este sistema de criação.

Palavras-chave: aves poedeiras, comportamento animal, condição ambiental, sistema de criação

\section{Influence of breeding systems on behavior of two lineages of laying hens exposed to two environmental conditions}

\begin{abstract}
This study aimed to evaluate the behavior and the welfare of hens comparing a conventional housing system (laying cages in battery) with another system using litter + nest. The effects of environmental conditions (stress or comfort) on behavior responses of laying hens were also analyzed by precision techniques. Two groups of 10 birds of Hy-line W36 and Hy-line Brown stains, at the beginning of the production phase, were submitted to two environmental conditions $\left(26^{\circ} \mathrm{C}\right.$ with $60 \% \mathrm{RU}$ or $35^{\circ} \mathrm{C}$ with $70 \% \mathrm{RU}$ ) and two housing systems (cages in battery or litter), during two weeks. The birds were marked with non-toxic paint and their behavior was evaluated by tape recorded by video cameras installed on the roof of the climatic chambers. Behavior parameters such as eating, drinking or dustbathing were recorded during 2 hours, in the morning and in the afternoon, in intervals of $1 \mathrm{~s}$ in order to investigate the frequency and lenght of each parameter. Housing system in litters allowed the expression of all natural behaviors of birds and those related to their comfort, which lead to improve the welfare conditions. The conventional housing system (laying cages) increased stress because birds tried to manifest their natural behavior in the absence of conditions for it
\end{abstract}

Key Words: laying hens, animal behavior, environmental conditions, housing systems

\section{Introdução}

Entre as mudanças no manejo e nos sistemas de criação de aves poedeiras que logo serão exigidas pela União Européia (EU) para atender às legislações de bem-estar animal, está a troca do atual sistema de bateria em gaiolas por um sistema que possibilite às aves a expressão de seus comportamentos naturais - utilizar ninho para postura, tomar banho de areia, empoleirar ou ainda bater e esticar as asas. Essas mudanças são estimuladas pelas exigências de um novo tipo de consumidor, preocupado com o bem-estar dos animais de produção e com a qualidade dos alimentos para o consumo.

Com base nessas tendências comerciais, serão necessárias alterações radicais nas instalações para poedeiras, além de mudanças na genética, visando à adequação das linhagens aos novos moldes do mercado. Todavia, segundo 
Becker (2002), deve-se considerar também que a criação intensiva exige a adaptação fisiológica e comportamental dos animais, o que exige estudos para avaliação dos sistemas de manejo mais adequados.

O grau de confinamento ao qual a ave poedeira é submetida é extremamente alto e impõe severas restrições ao seu comportamento. Normalmente, são usadas gaiolas que medem 30 a $35 \mathrm{~cm}$ de largura e $43 \mathrm{~cm}$ de comprimento para comportar 3 a 5 aves. Nessas condições, as aves não podem esticar as asas, mover-se livremente ou levantar-se totalmente no fundo da gaiola (o piso da gaiola é inclinado para que o ovo role em direção à calha coletora) (Singer, 1991).

Grande parte do comportamento normal da ave é impossibilitada pelo engaiolamento. O comportamento de acasalamento, incubação e cuidado com os pintinhos é impedido, preservando-se como única compulsão reprodutiva a atividade de pôr ovos. As aves não podem ciscar, empoleirar, andar livremente, tampouco limpar suas penas e se "sujar" com terra (Singer, 1991).

A comparação de estudos de comportamento de aves selvagens e domesticadas em ambientes controlados pelo homem indica que o repertório comportamental das aves em ambientes não-confinados geralmente é preservado, ocorrendo mudanças apenas na freqüência e intensidade das características comportamentais (Craig, 1992).

A seleção genética de aves em confinamento não altera seu comportamento quando criadas soltas. Comparando a linhagem comercial ISA a aves caipiras, Sales et al. (2000) verificaram o mesmo padrão geral de comportamento entre as aves.

Outro importante comportamento que não pode ser modificado é o banho de areia; a seqüência deste comportamento é realizada mesmo quando as aves estão sobre o piso de arame das gaiolas. Olsson et al. (2002) investigaram a motivação para este comportamento em poedeiras e constataram que a experiência prévia poderia ser um fator importante.

Assim, realizou-se este trabalho com os objetivos de avaliar o comportamento e o bem-estar de aves em um sistema de criação em cama e ninho, em comparação a um sistema de criação convencional (bateria de gaiolas), e estudar a influência das condições ambientais (estresse e conforto) no comportamento das linhagens avaliadas.

\section{Material e Métodos}

O experimento foi realizado no período de 02 de agosto a 10 de setembro de 2004 em câmara climática nas dependências do Núcleo de Pesquisa em Ambiência (NUPEA) do Departamento de Engenharia Rural da ESALQ/USP. Dez aves de cada linhagem (Hy-line W36 e Hy-line Brown) foram separadas em dois sistemas de criação: cinco no sistema de criação em gaiola e cinco no sistema cama+ninho para cada condição de ambiente proposta.

As aves de cada linhagem, em cada sistema de criação, foram expostas a duas condições ambientais: conforto térmico $=$ temperatura de $26^{\circ} \mathrm{C} \pm 2^{\circ} \mathrm{C}$ e umidade relativa de $60 \% \pm 2 \%$; estresse térmico $=$ temperatura de $35^{\circ} \mathrm{C} \pm 2^{\circ} \mathrm{C}$ e umidade relativa de $70 \% \pm 2 \%$.

Todas as aves foram expostas a cada uma das condições ambientais durante 14 dias consecutivos, em ambiente controlado, de acordo com Sevegnani etal. (2001), porém com período de adaptação gradativa ao ambiente, totalizando 21 dias de exposição ao ambiente controlado para cada tratamento.

As características de cada sistema de criação e a densidade de aves em cama e gaiola podem ser observadas na Tabela 1.

Os dados referentes à temperatura de bulbo seco (Tbs) e à umidade relativa (UR) foram coletados no interior da câmara climática por meio de miniestações meteorológicas e data logger $\mathrm{HOBO}^{\circledR}$. As mini-estações meteorológicas foram instaladas, respectivamente, a 1,70; 1,50 e 0,50 m de altura em relação ao piso da câmara e as leituras foram realizadas a cada 15 minutos ao longo das 24 hora, o que possibilitou traçar um perfil de temperatura dentro da câmara climática.

Para caracterizar a zona de conforto térmico adequada às aves, foi determinado o índice de conforto térmico entalpia, por meio da fórmula descrita por Villa Nova (1999), citado por Furlan (2001):

$$
H=6,7+0,243 * T b s+\left\{\frac{U R}{100} * 10^{\frac{7,5 * T b s}{237,3+T b s}}\right\}
$$

em que: $\mathrm{H}=$ entalpia $(\mathrm{kcal} / \mathrm{kg}$ ar seco); $\mathrm{Tbs}=$ temperatura ambiente (bulbo seco $)\left({ }^{\circ} \mathrm{C}\right)$ e UR $=$ umidade relativa do $\operatorname{ar}(\%)$.

Tabela 1 - Caracterização dos sistemas de criação

Table 1 - Breeding systems characterization

\begin{tabular}{lc}
\hline $\begin{array}{l}\text { Dimensões da gaiola } \\
\text { (comp. x largura x altura), cm }\end{array}$ & $50 \times 45 \times 40$ \\
Cage dimension (cm) & \\
Dimensões do boxe com cama (cm) & 5 \\
Box dimensions (cm) & \\
№ de aves por boxe ou gaiola & 450 \\
Birds/box or cage & \\
Área de gaiola para ave $\left(\mathrm{cm}^{2}\right)$ & 2.000 \\
$\begin{array}{l}\text { Cage area/bird }\left(\mathrm{cm}^{2}\right) \\
\text { Área de cama para ave }\left(\mathrm{cm}^{2}\right)\end{array}$ & 20 \\
$\begin{array}{l}\left.\text { Box area/bird (cm }{ }^{2}\right) \\
\text { Poleiro (cm/ave) - somente nos boxes }\end{array}$ & 2 \\
Perch (cm/bird) - only in box & \\
№ de bebedouros tipo Nipple para gaiola e boxe & \\
Nipple/cage or box & \\
№ de ninhos por boxe & \\
Nest/box &
\end{tabular}


Para acompanhamento e análise do comportamento, todas as aves foram identificadas por meio de um sistema de marcação individual (pintura do dorso com tinta atóxica), segundo Rudkin \& Stewart (2003). As imagens foram registradas por câmeras de vídeo instaladas no teto da câmara climática e ligadas a um microcomputador equipado com placa de captura de imagens e software para análise.

As imagens foram registradas a cada segundo, durante 1 hora pela manhã (de 10 às $11 \mathrm{~h}$ ) e 2 horas no período da tarde (de 13 às $14 \mathrm{~h}$ e de 16 às $17 \mathrm{~h}$ ), por dois dias consecutivos, para cada condição de ambiente e sistema de criação proposto. As imagens, gravadas a cada segundo, foram gerenciadas pelo software "VIDEOCAP 5.1" e armazenadas em um banco de dados. Durante a noite não foram registrados dados de comportamento.

Após o período de coleta, as imagens foram analisadas pelo método visual, ou seja, sem o auxílio de softwares de interpretação ou de análise de imagens, sendo analisadas para cada ave, cada comportamento e cada período do dia, sempre pela mesma pessoa.

Após as análises, foram calculadas as porcentagens de tempo de expressão de cada comportamento e o período, em minutos e segundos, de duração de cada comportamento.

Os padrões comportamentais foram avaliados de acordo com Rudkin \& Stewart (2003), considerando-se as atividades desenvolvidas individualmente pelas aves.

Foram avaliados os seguintes padrões comportamentais:

a) comendo - quando a ave está se alimentando, identificado geralmente quando a ave se encontra com a cabeça no comedouro;

b) bebendo - quando a ave está bebendo água, geralmente caracterizado quando a ave está bicando o bebedouro tipo Nipple;

c) investigando as penas - comportamento não agressivo, em que a ave investiga com o bico suas próprias penas ou as de outras aves;

d) banho de areia - comportamento característico das aves, que envolve uma seqüência de ciscar e jogar "areia" sobre seu corpo, além de movimentos rápidos de sacudir as penas;

e) movimentos de conforto - comportamentos apresentados pelas aves em condição de conforto e bem-estar, como bater e esticar as asas e sacudir as penas;

f) ciscando - comportamento característico das aves, em que a ave exploram seu território com os pés e o bico;

g) empoleirar - por ser um comportamento natural, está associado ao bem-estar das aves, sendo verificado quando a ave sobe em algum aparato acima do nível do solo;

h) agressividade - relacionado à condição de se estabelecer dominância no grupo ou a situações de estresse, geral- mente caracterizado por bicadas rápidas e fortes em locais como a crista e outras partes da cabeça de outras aves;

i) sentada - quando a ave encontra-se sentada na cama ou no substrato;

j) procurando ninho - considerado pré-postura, esse comportamento é facilmente detectável, sendo caracterizado por entradas rápidas e freqüentes ao ninho na tentativa de avaliar o local antes de pôr os ovos;

k) postura - presença da ave sentada no ninho e com o ovo;

1) parada - quando a ave não apresenta nenhum movimento, ou aparentemente seu comportamento não se enquadra em nenhum dos anteriores.

\section{Resultados e Discussão}

Constam nas Figuras 1 e 2 o comportamento de entalpia $(\mathrm{KJ} / \mathrm{kg}$ ar seco) para as condições de conforto e estresse térmico e a respectiva faixa com os valores limites considerados ideais para aves.

Na Figura 1, nota-se que os valores médios de quantidade de energia existente na massa de ar seco estão dentro das faixas limites de conforto para o índice. Esse mesmo fato, no entanto, não é observado na Figura 2, em que a análise foi feita para a condição de estresse. Portanto, os valores da entalpia são superiores aos de conforto do índice, caracterizando condição de estresse térmico.

De acordo com Johnson \& Hogg (1996), monitorar aves tem algumas dificuldades particulares, em razão dos comportamentos às vezes imprevisíveis e muito rápidos, o que pode dificultar as análises.

Na comparação entre linhagens (Tabela 2), a Hy-Line Brown, no período da manhã, permaneceu mais tempo comendo, bebendo, ciscando, procurando ninho, tomando banho de areia e investigando penas que a linhagem HyLine W36. Por outro lado, as aves da linhagem Hy-Line W36 permaneceram mais tempo empoleiradas e em postura. No período da tarde, a diferença foi provocada pela expressão de comportamentos como sentar e ficar parada e a ocorrência de agressividade (somente na linhagem HyLine W36).

Ressalta-se que o comportamento de postura no período da tarde, como na parte da manhã, só foi constatado para a linhagem Hy-Line W36, provavelmente em razão da melhor adaptação desta linhagem à postura, comprovada pelo tempo de manifestação desse comportamento em relação à outra linhagem avaliada.

$\mathrm{Na}$ avaliação dos comportamentos para a condição de conforto e o sistema de criação cama+ninho, foi evidente também (Tabela 2) a expressão dos comportamentos de conforto pelas aves. 


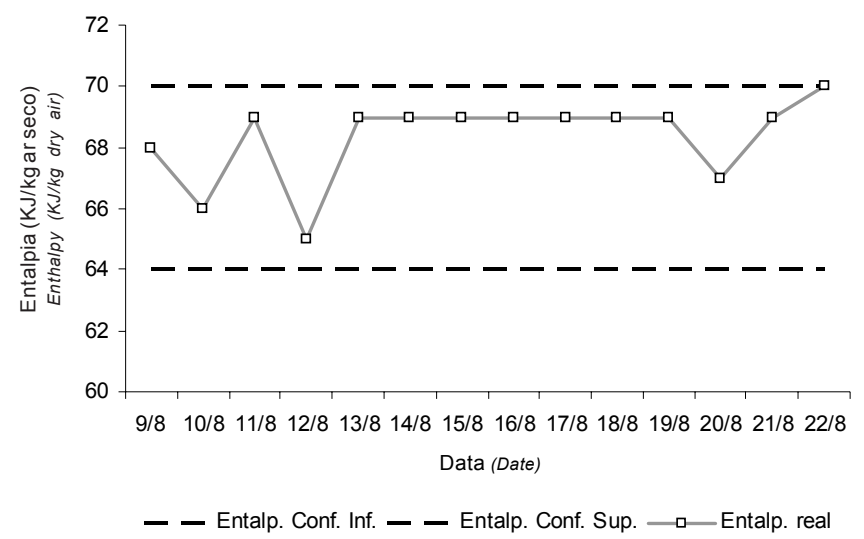

Figura 1 - Valores de entalpia em câmara climática para a condição de conforto (entalp. real) e a faixa considerada ideal para aves com os respectivos limites, inferior e superior, de conforto.

Figure 1 - Values of enthalpy within the climate chamber at comfort conditions (Real Enthalpy) and the inferior and superior limits of the ideal range of comfort for birds.

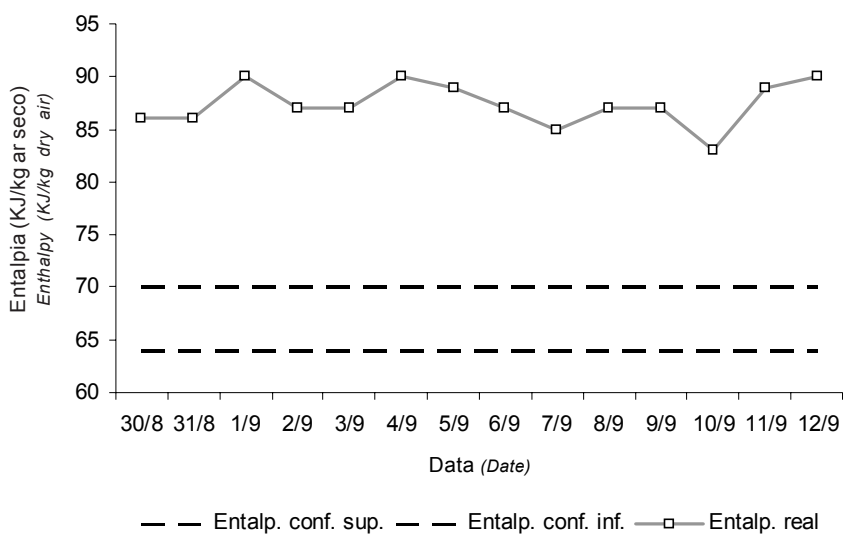

Figura 2 - Valores de entalpia em câmara climática para a condição de estresse (entalp. real) e a faixa considerada ideal com os respectivos limites, inferior e superior, de conforto.

Figure 2 - Values of enthalpy within the climate chamber at stress conditions (Real Enthalpy) and the inferior and superior limits of the ideal range of comfort for birds.

Tabela 2 - Valores percentuais de tempo médio em que as aves expressaram seus comportamentos na condição de conforto térmico Table 2 - Values of average time (in percentage) spent by birds to express their behaviors at thermal comfort condition

\begin{tabular}{|c|c|c|c|c|c|c|}
\hline \multirow{3}{*}{$\begin{array}{l}\text { Padrão de comportamento } \\
\text { Behavior }\end{array}$} & \multicolumn{6}{|c|}{$\begin{array}{l}\text { Condição de conforto } \\
\text { Comfort condition }\end{array}$} \\
\hline & \multicolumn{2}{|c|}{$\begin{array}{l}\text { Período da manhã } \\
\text { Morning period }\end{array}$} & \multicolumn{2}{|c|}{$\begin{array}{l}\text { Período da tarde } \\
\text { Afternoon period }\end{array}$} & \multicolumn{2}{|c|}{ Total } \\
\hline & ly-Line Brown & Hy-Line W36 & Hy-Line Brown & Hy-Line W36 & Hy-Line Brown & Hy-Line W36 \\
\hline Comendo (Eating) & 32 & 28 & 23 & 20 & 27,5 & 24 \\
\hline Proc. ninho (Looking for nest) & 21 & 13 & 12 & 0,4 & 16,5 & 6,7 \\
\hline Postura (Laying) & - & 26 & - & 18 & - & 22 \\
\hline Mov. conforto (Comfort moviments & $n t s)$ & 1 & 1 & 1 & 1 & 1 \\
\hline Invest. penas (Feather pecking) & 6 & 4 & 7 & 7 & 6,5 & 5,5 \\
\hline Empoleirar (Perching) & - & 15 & 9 & 19 & 4,5 & 17 \\
\hline Banho de areia (Dustbathing) & 7 & - & 9 & 4 & 8 & 2 \\
\hline
\end{tabular}

Um fator de grande influência na expressão dos comportamentos das aves é o horário, visto que todos os animais seguem um biorritmo. Este biorritmo está ligado principalmente ao fotoperíodo, que, neste período foi de $16: 8$, ou seja, 16 horas de luz ( 8 às $24 \mathrm{~h}$ ) e 8 horas de escuro. Deve-se, portanto, considerar a influência do período de avaliação (manhã e tarde) na expressão dos comportamentos, ou seja, no período da manhã, as aves passaram mais tempo no comedouro (aproximadamente $30 \%$ ), certamente por causa do horário de araçoamento. Outros comportamentos que também merecem destaque no período da manhã são o de ciscar e o de procurar o ninho, além do comportamento de empoleirar, manifestado em tempo considerável (15\%) pela linhagem Hy-Line W36.

No período da tarde, houve ligeira redução no comportamento de comer e aumento nos comportamentos de investigar penas, empoleirar, tomar banho de areia e ficar parada. Além destes comportamentos, apareceu também o comportamento de agressividade.

Em análise geral da Tabela 2, nota-se que, para a condição de conforto e o sistema de criação em cama, as linhagens não apresentaram diferenças quanto à expressão dos comportamentos avaliados, tanto para o período da tarde quanto para o da manhã. 
Tabela 3 - Tempo médio, em porcentagem, em que as aves expressaram seus comportamentos na condição de estresse térmico Table 3 - Average time (in percentage) spent by birds to express their behaviors at heat stress condition

\begin{tabular}{|c|c|c|c|c|c|c|c|}
\hline \multirow{3}{*}{$\begin{array}{l}\text { Padrão de comportamento } \\
\text { Behavior }\end{array}$} & \multicolumn{7}{|c|}{$\begin{array}{l}\text { Condição de estresse } \\
\text { Stress condition }\end{array}$} \\
\hline & \multicolumn{3}{|c|}{$\begin{array}{l}\text { Período da manhã } \\
\text { Morning period }\end{array}$} & \multicolumn{2}{|c|}{$\begin{array}{l}\text { Período da tarde } \\
\text { Afternoon period }\end{array}$} & \multicolumn{2}{|c|}{ Total } \\
\hline & Hy-Lit & e Brown & Hy-Line W36 & Hy-Line Brown & Hy-Line W36 & Hy-Line Brown & Hy-Line W36 \\
\hline Comendo (Eating) & & 20 & 36 & 16 & 25 & 18 & 30,5 \\
\hline Proc. ninho (Looking for nest) & & - & - & - & 25 & - & 12,5 \\
\hline Postura (Laying) & & - & - & - & - & - & - \\
\hline Mov. conforto (Comfort movim & nents) & - & - & - & - & - & - \\
\hline Invest. penas (Feather pecking) & & 3 & 4 & 5 & 5 & 4 & 4,5 \\
\hline Empoleirar (Perching) & & 24 & - & 19 & 4 & 21,5 & 2 \\
\hline Banho de areia (Dustbathing) & & - & - & - & - & - & - \\
\hline
\end{tabular}

As aves sob condição de estresse térmico, no sistema de criação cama+ninho, em comparação àquelas em conforto, não apresentaram, ou apresentaram pouco, os chamados movimentos de conforto. Outra característica da situação de estresse foi o aumento no comportamento de beber água (Sturkie, 1967) e a redução brusca no consumo de alimentos (Payne, 1967). Também foi expressivo o aumento dos comportamentos de sentar e ficar parada, certamente medidas comportamentais que influenciam diretamente a fisiologia dos animais, de modo a não aumentar a produção de calor pelos movimentos.

Na Tabela 3 observa-se o comportamento das aves para a situação de estresse térmico e o sistema de criação em cama.

Em condições de estresse térmico, a linhagem Hy-Line W36 passou mais tempo comendo (no comedouro) que a Hy-Line Brown, tanto no período da manhã quanto à tarde, o que não indica necessariamente que a linhagem Hy-Line W36 tenha consumido mais ração, visto que esse comportamento se caracteriza pela presença da ave no comedouro e não necessariamente pela ação de se alimentar.

Outro ponto importante é o tempo em que as aves da linhagem Hy-Line Brown permaneceram paradas (24\%) em relação às da linhagem Hy-Line W36, o que evidencia que esta linhagem é menos resistente às condições de estresse térmico.

No período da tarde, as aves da linhagem Hy-Line Brown mantiveram-se por menos tempo empoleiradas, enquanto as da linhagem Hy-Line W36 passaram a expressar este comportamento.

Os comportamentos de conforto, como banho de "areia" e movimentos de conforto, não ocorreram na condição de estresse, tanto no período da manhã como no da tarde, o que confirma os efeitos do estresse provocados por altas temperaturas e pela umidade relativa na expressão dos comportamentos normais das aves.

Para melhor discussão dos percentuais de expressão dos comportamentos de cada linhagem e de cada condição de criação, tornam-se necessários os seguintes comentários sobre os comportamentos avaliados:

comer - no sistema em gaiolas, foi possível notar aumento deste comportamento, característico de animais em confinamento (Huges \& Duncan, 1988), que não têm outra alternativa para passar o tempo. Talvez o sistema de criação em cama, por proporcionar ambiente mais rico e oferecer outras opções além de comer, possa ter contribuído para esta diferença, haja vista a maior distribuição nas porcentagens de tempo gasto em outras atividades;

beber - as diferenças nas porcentagens deste comportamento podem também ser explicadas, em parte, pela falta de enriquecimento do ambiente, ou seja, a gaiola é um ambiente com poucos atrativos às aves e um dos poucos, senão o único, é o bico do bebedouro tipo Nipple, que, muitas vezes, é utilizado não somente para saciar a sede das aves, mas também como uma "distração" em meio a uma condição muito propícia à ocorrência de estresse;

investigar penas - por ser natural das aves, esse comportamento aparece em todas as condições ambientais e em todos os sistemas de criação, variando somente em intensidade de execução. Esse comportamento de bemestar das aves é muito importante, pois, a partir dele, as aves ou organizam suas penas e espalham o óleo proveniente da glândula uropigiana; 
banho de areia - comprovando o que foi verificado por Appleby et al. (1993), este é um comportamento que as aves apresentam ou tendem a apresentar sob qualquer condição de criação, sendo observado até mesmo no sistema em gaiolas, onde não há espaço nem material de cama, podendo ocasionar frustração, causando estresse e gerando comportamentos fora dos padrões.O sistema em cama favoreceu a expressão e o maior período de duração deste comportamento, pois possuía uma área com cama, atraindo as aves e aumentando o período de expressão deste comportamento. Segundo Petherick et al. (1995), a cama exerce um estímulo visual muito importante às aves, constituindo-se um dos principais fatores que desencadeiam os processos do comportamento de banho de areia. Segundo Vestergaard (1982), o tempo de duração do comportamento de banho de areia é, em média,de 27 minutos. Este valor não foi comprovado neste estudo, visto que o comportamento de banho de areia, em condições de conforto e sistema de criação em cama, foi de aproximadamete 8 minutos e não foi verificado durante o período de estresse térmico, o que indica o abandono desse comportamento como recurso para não aumentar a produção de calor por meio de atividades físicas;

movimentos de conforto - no sistema de criação em gaiolas, a expressão destes movimentos torna-se crítica, pois as aves são mantidas em confinamento e a altas densidades, o que as impede de esticar as asas ou chacoalhar as penas. Segundo Dawkins \& Hardie (1989), citados por Rudkin \& Stewart (2003), é necessário que haja uma área de no mínimo $860 \mathrm{~cm}^{2}$ para que comportamentos como bater as asas possam ocorrer naturalmente. Como a gaiola utilizada neste trabalho não cumpre todos estes requisitos (Tabela 1), comportamentos como esse acabam prejudicados, pois, embora a ave tente executá-los, não consegue por causa das dimensões da gaiola, o que, na maioria das vezes, leva os animais à frustração e ao estresse;

ciscar - no sistema de criação em gaiolas, as aves, apresentaram, mesmo sem a presença de nenhum substrato, o comportamento de ciscar o fundo das gaiolas. Como este é um comportamento natural das aves, a frustração pelo impedimento deste comportamento provavelmente contribuiu para o agravamento do estresse;

empoleirar - durante as análises de imagens para este comportamento, foi possível verificar também que outros comportamentos foram executados simultaneamente ao de empoleirar, ou seja, comportamentos como investigar penas, movimentos de conforto e bater e esticar as asas, puderam ser constatados simultaneamente ao de empoleirar durante a condição térmica de conforto. Para a condição de estresse térmico, o comportamento mais recorrente juntamente ao de empoleirar foi o de beber água, o que também foi verificado por Rudkin \& Stewart (2003), que classificaram-no como estereótipo de determinada ave a determinado bico do bebedouro nipple.

agressividade - no sistema de criação em gaiola, constatouse que, mesmo depois de estabelecida uma dominância e hierarquia dentro do grupo, as aves continuaram apresentando agressividade, provavelmente um indicativo da maior carga de estresse no sistema de criação em confinamento. Ressalta-se também que não foram observados problemas de agressividade decorrentes do tipo de marcação utilizado nas aves;

sentada - Rutz (1994) relatou que, em condição ambiental acima da zona termoneutra, a atividade física das aves foi reduzida, como forma de diminuir a produção interna de calor, de modo que as aves passavam mais tempo sentadas. Segundo Appleby et al. (1998) e Keeling (1995), aves criadas em sistemas com cama ou em semiconfinamento tendem a ficar menos tempo sentadas ou paradas, em razão da maior disponibilidade de recursos nesses sistemas, favorecendo seu bem-estar;

pré-postura e postura - autores como Wood-Gush (1975), Appleby et al. (1992) e Petherick et al. (1994) relataram que o comportamento de pré-postura ou de procurar o ninho é caracterizado pelo aumento rápido nas atividades locomotoras e exploratórias das aves, que avaliam de modo relativamente rápido os locais com potenciais chances para a postura. No sistema convencional de criação em gaiolas, o comportamento de pré-postura é bem diferente do apresentado em condições de criação em cama com ninho, principalmente porque as aves não possuem ninhos nem espaço para iniciar um comportamento exploratório, como aquele realizado pelas aves soltas. Neste caso, as aves criadas em gaiolas tendem a apresentar pouco ou nenhum comportamento de pré-postura e, quando apresentam, é quase imperceptível, pois ocorre de forma muito rápida e pouco expressiva (Duncan et al., 1978). Ocomportamento de postura em ninho foi preferencial, pois ocorreu em praticamente $100 \%$ das aves criadas no sistema cama + ninho, comprovando que se adaptaram bem às condições do ninho e "aprenderam" a utilizá-lo durante o período de adaptação às condições da câmara climática, resultando em pouca ocorrência de ovos postos em cama durante o período de adaptação e na ausência de ovos postos na cama durante o período de coleta de dados.

parada - no sistema de criação em gaiolas, esse comportamento pode ser resultante, além do estresse térmico, de aspectos como agressividade, por fatores como dominância ou disputa por espaço ou, ainda, pelo estresse provocado pelo alto grau de confinamento das aves. 
A análise dos comportamentos no sistema convencional de criação, ou seja, em gaiolas, foi mais difícil, em virtude de problemas como o posicionamento das câmeras de vídeo (instaladas no teto) e a quantidade de aves por gaiola (cinco aves/gaiola), que impediram a análise mais apurada das imagens, como aquela realizada para a condição de criação cama+ninho.

Outro problema foi a impossibilidade da completa avaliação individual das aves, agravada pela distância das câmeras de vídeo, pela aglomeração dos animais na gaiola e pelo tipo de marcação utilizado, que não foi muito eficiente para distinção dos animais.

Diante disso, as análises foram feitas no conjunto das aves nas gaiolas ou no conjunto de seus comportamentos para as condições de ambiente propostas.

Foi possível observar também, durante a análise das imagens, os padrões comportamentais das aves em gaiola seguiram uma seqüência pequena de comportamentos (comendo, bebendo, sentada e parada como mais freqüentes), ou seja, o próprio sistema de criação impediu que todos os comportamentos expressos pelas aves em cama fossem apresentados também pelas aves em gaiola.

Assim, em condições de conforto térmico, as aves das linhagens Hy-Line Brown e Hy-Line W36, nos períodos da manhã e tarde, passaram a maior parte do tempo comendo, podendo ser evidenciados outros comportamentos como o de investigar penas e beber água.

$\mathrm{Na}$ condição de estresse térmico, os comportamentos detectados com maior freqüência, principalmente no período da tarde, foram os de sentar, ficar parada e beber água, característicos de aves sob condições de estresse. O comportamento de comer também reduziu significativamente $(50 \%)$, principalmente na linhagem Hy-Line Brown.

Comportamentos como movimentos de conforto, como postura e pré-postura, foram constatados, mas não foram contabilizados nas análises, pois não foram registrados o início e fim de sua expressão.

\section{Conclusões}

No sistema de criação em cama, verificou-se a ocorrência dos comportamentos naturais de conforto, possivelmente em razão das melhores condições de bem-estar em comparação ao sistema de criação em gaiolas, no qual esses comportamentos não ocorreram.

Verificou-se nítida preferência pela postura em ninho nas duas linhagens avaliadas, tanto para a condição de conforto quanto para a condição de estresse térmico, não se constatando, portanto, influência das condições ambientais no uso do ninho pelas aves.
No sistema de gaiolas, as aves tentaram constantemente executar seus hábitos naturais, como ciscar, ou o comportamento de pré-postura e de procurar o ninho, o que certamente agravou a condição de estresse, haja vista a impossibilidade de expressão destes comportamentos nesse sistema de criação.

\section{Literatura Citada}

APPLEBY, M.C.; SMITH, S.F.; HUGHES, B.O. Nesting, dustbathing and perching by baying hens in cages: effects of design on behaviour and welfare. British Poultry Science, v.34, p.835$847,1993$.

APPLEBY, M.C. Modification of laying hen cages to improve behaviour. Poultry Science, v.77, p.1828-1832, 1998.

BECKER, B.G. Comportamento das aves e sua aplicação prática. In: CONFERÊNCIA APINCO DE CIÊNCIA E TECNOLOGIA AVÍCOLAS, 2002, Campinas. Anais... Campinas: Fundação APINCO de Tecnologia e Ciência Avícolas, 2002. p.81-90.

CRAIG, J.V. Measuring social behavior in poultry. Poultry Science, v.71, p.650-657, 1992

DUNCAN, I.J.H.; SAVORY, C.J.; WOOD-GUSH, D.G.M. Observations on the reproductive behaviour of domestic fowl in the wild. Applied Animal Ethology, n.4, p.29-42, 1978.

FURLAN, R.A. Avaliação da nebulização e abertura de cortinas na redução da temperatura do ar em ambiente protegido. Piracicaba: Escola Superior de Agricultura Luiz de Queiroz, 2001. 146p. Tese (Doutorado em Irrigação e Drenagem) - Escola Superior de Agricultura Luiz de Queiroz, 2001.

HUGHES, B.O.; DUNCAN, I.J.H. The notion of ethological "need", models of motivation and animal welfare. Animal Behaviour, n.36, p.1696-1707, 1988

JOHNSON, N.; HOGG, D. Learning the distribution of object trajectories for evente recognition. Image and Vision Computing, v.14, n.3, p.609-615, 1996.

KEELING, L. Spacing behaviour and an ethological approach to assessing optimun space allocations for groups of laiyng hens. Applied Animal Behavior Science, v.44, p.171-186, 1995

OLSSON, I.A.S.; KEELING, L.J. Night time roosting in laying hens and the effect of thwarting access to perches. Applied Animal Behavior Science, v.68, p.243-256, 2000.

PAYNE, G.C. Environmental temperature and egg production. In: HORTON-SMith, C.; AMBROSE, E.C. (Eds.) The physiology of the domestic fowl. Scotland and London, Edinburgh: Oliver and Boyd, 1967. p.235-241.

PETHERICK, J.C.; SEAWRIGHT, E.; WADDINGTON, D Influence of quantity of litter on nest box selection and nesting behaviour of domestic hens. British Poultry Science, n.34, p.857-872, 1994 .

PETHERICK, J.C.; SEAWRIGHT, E.; WADDINGTON, D. et al. The role of perception in the causation of dustbathing behaviour in domestic fowl. Animal Behaviour, n.49, p.1521-1530, 1995

RUDKIN, C.; STEWART, G.D. Behaviour of hens in cages - a pilot study using video tapes. A Report for the Rural Industries Researsh and Development Corporation (RIRDC), v.40, n. 477, p. $102,2003$.

RUTZ, F. Aspectos fisiológicos que regulam o conforto térmico das aves. In: CONFERÊNCIA APINCO DE CIÊNCIA E TECNOLOGIA AVÍCOLAS, 1994, Santos. Anais... Santos: Fundação Apinco de Ciência e Tecnologia Avícolas, 1994. p.99-110.

SALES, M.N.G.; GARCIA, R.A.M.; PINHEIRO MACHADO, F.L.C. et al. Isa Brown and native Brazilian chicks raised on pasture display similar behavior. In: INTERNATIONAL CONGRESS OF THE ISAE, 34., 2000, Florianópolis. Proceedings... Florianópolis: 2000. p.57. 
SEVEGNANI, K.B.; MACARI, M.; NÄÄS, I.A. et al. Variação da temperatura média corporal de frangos de corte em terminação, submetidos às temperaturas de $24 \mathrm{e} 32^{\circ} \mathrm{C}$, em câmara climática. Revista Brasileira de Ciência Avícola, supl. 3, p.58, 2001 b.

SINGER, P. Animal liberation. rev.ed. New York: Harper Perennial - Avon Books, 1991. 320p.

STURKIE, P.D. Fisiologia aviaria. Zaragoza: Acribia, 1967. 607p. VESTERGAARD, K. Dustbathing in the domestic fowl - diurnal rhythm and dust deprivation. Applied Animal Ethology, n. 8, p.487-495, 1982 .
WOOD-GUSH, D.G.M. Nest construction by the domestic hen: some comparative and physiological considerations. In: WRIGHT, P.; CARYL, P.G.; VOWLES, D.M. (Eds). Neural and endocrine aspects of behavior in birds. Amsterdam: Elsevier, 1975. p.35-49.

Recebido: 26/04/05

Aprovado: 23/01/06 